\title{
Hydrogen Outgassing and Surface Properties of TiN-Coated Stainless Steel Chambers*
}

\author{
P. He ${ }^{\dagger}$ H.C. Hseuh, M. Mapes, R. Todd, D. Weiss and D. Wilson \\ Collider-Accelerator Department, Brookhaven National Laboratory, Upton, NY 11973, USA
}

\begin{abstract}
The stainless steel vacuum chambers of the $248 \mathrm{~m}$ accumulator ring of Spallation Neutron Source (SNS) are coated with $\sim 100 \mathrm{~nm}$ of titanium nitride (TiN) to reduce the secondary e lectron yield. The coating is produced by DC magnetron sputtering using a long cathode imbedded with permanent magnets. The outgassing rates of several SNS half-cell chambers were measured with and without TiN coating, and before and after in-situ bake. One potential benefit of a TiN coating is to serve as hydrogen permeation barrier that reduces the ultimate outgassing rate. By varying the coating parameters, films of different surface roughness were produced and analyzed by Auger electron spectroscopy, scanning electron microscopy and atomic force microscopy to illustrate the dependence of the outgassing on the film structure.
\end{abstract}

\section{INTRODUCTION}

The inner surface of the SNS ring vacuum chambers are coated with $\sim 100 \mathrm{~nm}$ of titanium nitride (TiN). This is to minimize the secondary electron yield (SEY) from the chamber wall, and thus avoid the so-called e-p instability caused by electron multi-pacting as o bserved in a few high-intensity proton and positron storage rings. The required average operating pressure for the SNS storage ring is $5 \times 10-9$ Torr. The hydrogen outgassing $r$ ate of stainless steel, based on theory and practice, is generally well understood and confident estimates can be made for a wide variety of conditions. However, because of the required TiN coating to reduce SEY, accurate outgassing data for coated chambers is necessary to confirm vacuum simulations.

It has been reported in the literature that TiN coatings on stainless steel substrates act as a hydrogen permeation barrier resulting in a reduction in hydrogen outgassing [1]. The work presented herein determines if this barrier is present, and to what degree. The outgassing properties of TiN coating are strongly dependent on the deposition method and the process parameters for the given method. Reactive sputtering method has been widely used to prepare TiN films. The properties of TiN films prepared by reactive sputtering depend on the process parameters, such as composition of the gas mixture, gas pressure, substrate temperature, etc.[2][3]. The effects of various treatments on a stainless

\footnotetext{
"Work performed under the auspices of the U.S. Department of Energy

† Corresponding author email: phe@bnl.gov
} 
steel surface have been extensively studied. In this work, the structure of the TiN films was analyzed by Auger Electron Spectroscopy (AES), Scanning Electron Microscopy (SEM) and Atomic Force Microscopy (AFM) to clarify the dependence of the barrier effect on the film structure. The outgassing rates of the stainless steel chambers with and without the TiN film were measured and correlated with the film structure.

\section{TIN COATING OF SNS RING VACUUM CHAMBERS}

\section{SNS Ring Vacuum Chamber}

The SNS ring has a circumference of $248 \mathrm{~m}$. It consists of 4 arc sections of $34 \mathrm{~m}$ each and 4 straight sections of $28 \mathrm{~m}$ each. There are 32 arc half cell chambers of four types varying slightly from one to the others [4]. Each SNS chamber, constructed of either $304 \mathrm{LN}$ or $316 \mathrm{~L}$ stainless steel, is approximately $4 \mathrm{~m}$ long and over $20 \mathrm{~cm}$ in diameter as shown schematically in Figure 1.

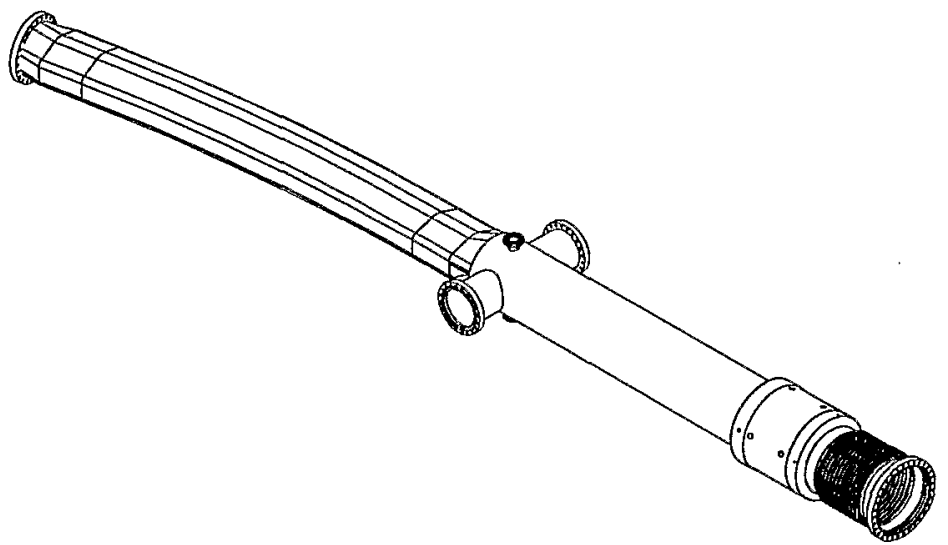

FIGURE 1. Typical SNS ring half-cell chamber consisting dipole section, pumping ports, quadrupole chamber section, beam position monitor and bellows.

\section{TiN Coating Processing}

The TiN coatings are deposited by the $\mathrm{DC}$ reactive magnetron sputtering method [5]. Figure 2 shows the sputter coating system for our chambers. The system has a water-cooled coaxial magnetron sputter source of $38 \mathrm{~mm}$ in diameter. The maximum transverse component of the magnetic field at the face of the titanium target is about 1000 Gauss. A variable power supply of $10 \mathrm{KW}$ maximum is used as the power source. High purity argon and nitrogen are used as the sputtering and reactive gases, respectively.

The vacuum chambers are evacuated by a rotary mechanical pump and a turbopump. Both argon inert gas flow and nitrogen reactive gas flow are regulated using mass flow controllers. The pressure in the chamber is monitored 
by a capacitance manometer. Prior to coating the chambers are degassed in a vacuum furnace at a practical upper limit of $450^{\circ} \mathrm{C}$ for 48 hours to remove hydrogen from the bulk. All coatings have been done with the chamber at a temperature of $250^{\circ} \mathrm{C}$. The sputtering chamber pressure is usually reduced to $5 \times 10^{-7}$ Torr before deposition. Then the argon was introduced and the target was pre-sputtered in a pure argon atmosphere for about 10 minutes, in order to lay down a Ti underlayer which helps to control the residual stress produced by the deposition process, and improve the adhesion of the coating. After presputtering, the nitrogen is introduced into the chamber through a $6 \mathrm{~mm}$ diameter Ti tube which runs the length of the cathode. Small holes located every $15 \mathrm{~cm}$ on the $\mathrm{Ti}$ tube provide even distribution of reactive gas, which is necessary for obtaining a uniform longitudinal coating. The reactive process is controlled using partial pressure feedback of nitrogen gas. Figure 3 shows the discharge plasma during coating. The sputtering conditions are listed in Table 1.

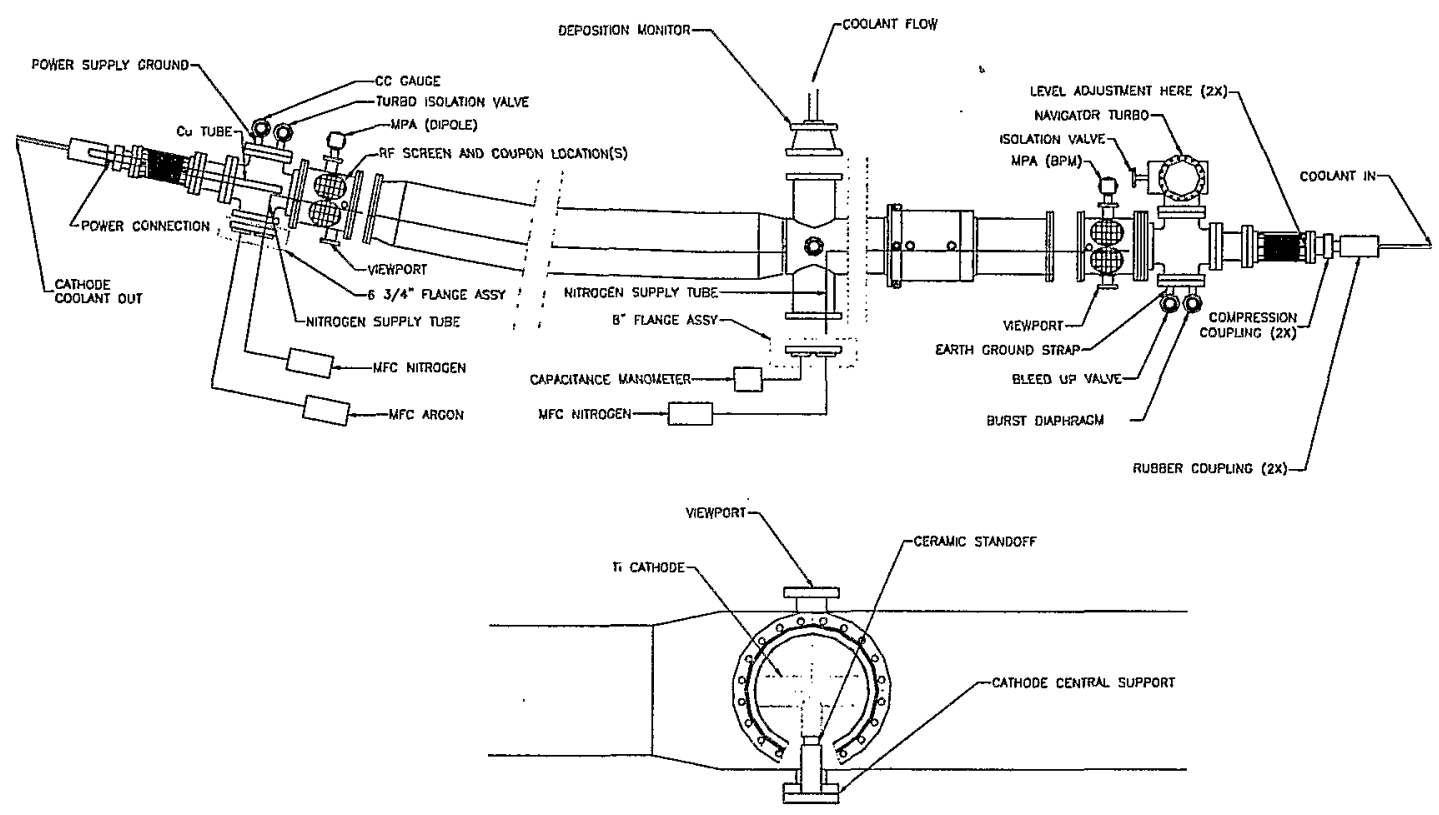

FIGURE 2. Schematic of magnetron sputtering coating setup for SNS ring halfcell chambers

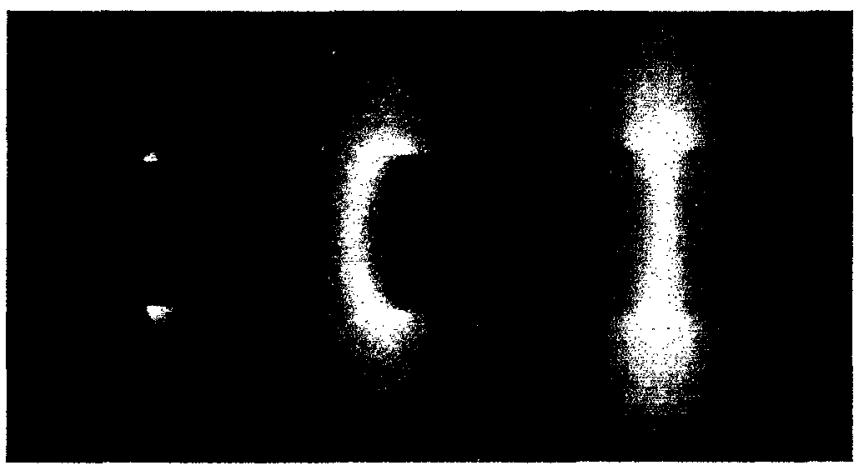

FIGURE 3. Discharge characteristics of DC magnetron sputtering in SNS ring halfcell chamber. 
It has been shown in the literature, and in our measurements, that the surface roughness of the films has a direct impact on the SEY of the film. For this reason, chambers have been coated at both high and low pressure to study this effect, as the low SEY is our driving parameter. Additionally, some TiN films produced at low pressure were also subjected to glow discharge treatment (GDT) subsequent to coating using an argon current of $1 \mathrm{~A}$ at $325 \mathrm{~V}$ for 5 minutes, for an accumulated dosage of $\sim 10^{17} \mathrm{ion} / \mathrm{cm}^{2}$. GDT was found to be of little benefit in reducing the outgassing chambers coated at high pressure, and in both high and low pressure coatings, even increased the measured SEY.

TABLE 1. Deposition Conditions for SNS Ring Vacuum Chambers

\begin{tabular}{lcc} 
& High Pressure (HP) Coating & Low Pressure (LP) Coating \\
\hline Total Pressure (mTorr) & 5.0 & 1.5 \\
Cathode Potential(V) & 300 & 350 \\
Cathode Current(A) & 10 & 10 \\
Argon Gas Flow(sccm) & 15.5 & 5.2 \\
Nitrogen Gas Flow(sccm) & $4.1,1.6$ & $4.6,2.3$ \\
Chamber Temperature $\left({ }^{\circ} \mathrm{C}\right)$ & $200 \sim 250$ & $200 \sim 250$ \\
Film Thickness(nm) & $200 \sim 400$ & $200 \sim 400$ \\
\hline
\end{tabular}

\section{SURFACE ANALYSIS AND OUTGASSING MEASUREMENT}

After deposition, the stainless steel witness coupons (coated together with the chambers) are sent to Brookhaven's Material Science Department for surface analysis. The concentrations of elements on the surface are determined using AES, while surface topology and cross-section structure of the films are observed by means of SEM and AFM.

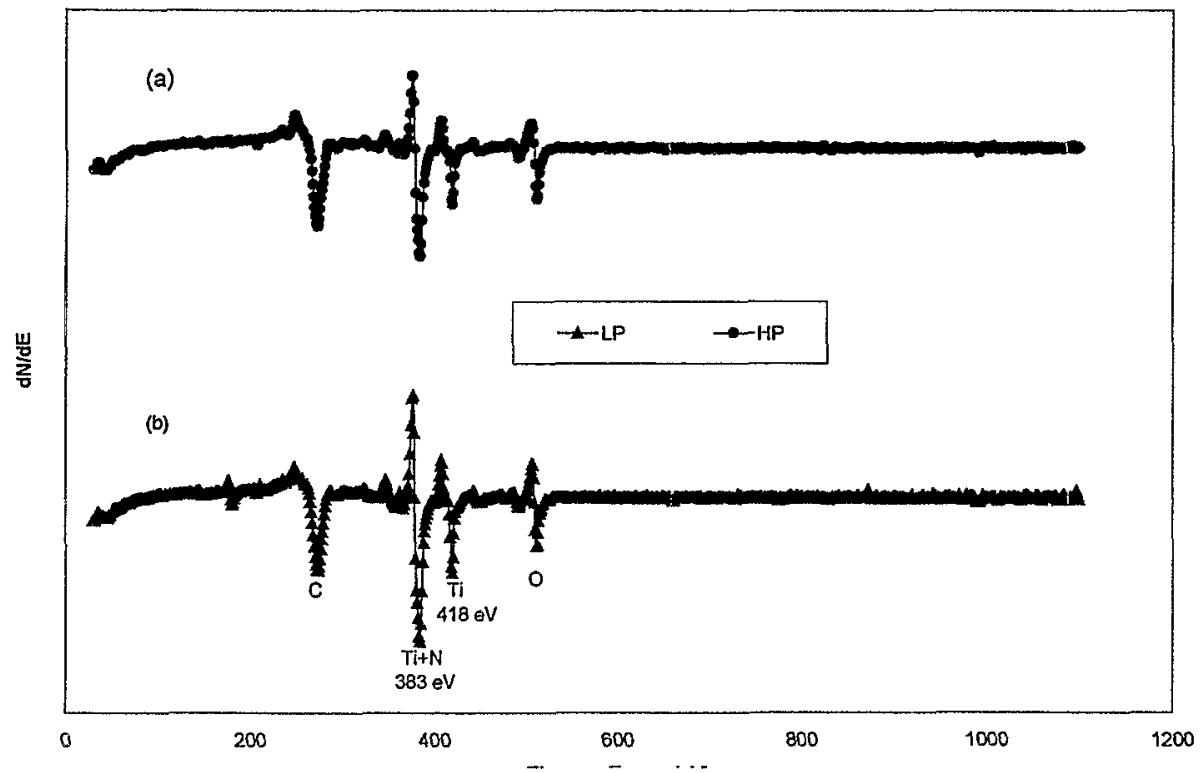

FIGURE 4. Auger spectra of TiN film after air expossure: (a) HP Coating; (b) LP Coating. 


\section{TiN film Surface Characteristics}

\section{AES Spectra}

The shapes of the Auger peaks for Ti+N (@383eV) and Ti (@418eV) are similar with coatings produced at both high and low pressures. The peaks for Ti and $\mathrm{N}$ are higher for the LP sample. This is likely a result of the LP sample having reduced surface roughness with a corresponding increase in SEY. However the stoichiometric ratio of both films is similar.

\section{SEM Micrographs}

The SEM microphotographs of the surface and cross-section of the TiN films prepared at two sputtering pressures show that the film prepared at low sputtering pressure has the fibrous structure of the zone T-type and the film prepared at high sputtering pressure has a voided structure of the zone I-type, according to the Thornton classification [6]. Additionally, high pressure films were found to be hydroscopic as a result of porosity and exhibited poor initial outgassing prior to in-situ bakeout.

(a)

Brown color

Coated - @ 5 mTorr,w/o GDT
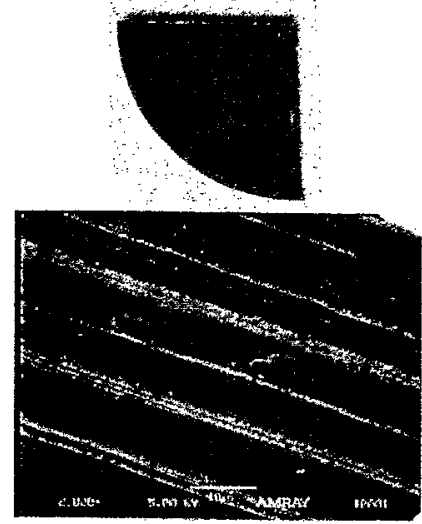

plan view

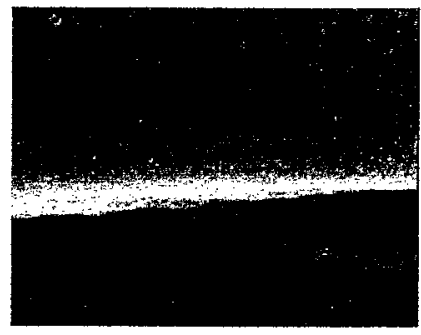

cross-section (b)

Gold color

- @ 1.5 mTorr, w/ Ar GDT
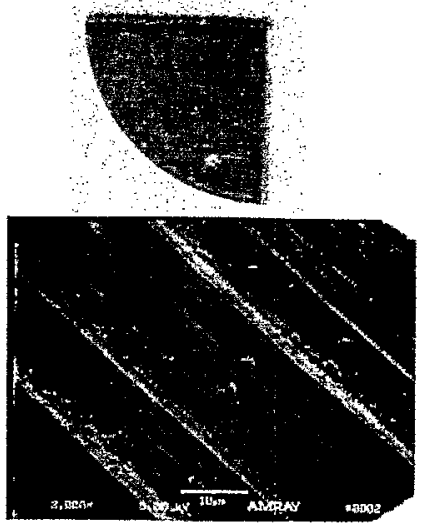

plan view

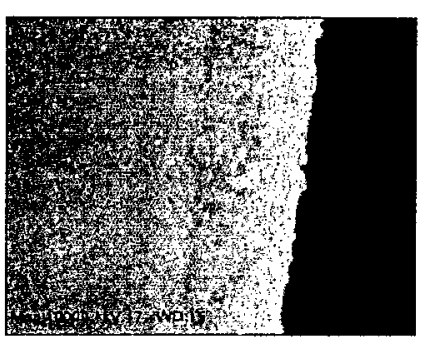

cross-section

FIGURE 5. SEM Images: (a) High Pressure Coating; (b) Low Pressure Coating 


\section{AFM Images}

To correlate and confirm the SEM results, AFM imaging was performed on identical coupons. While the AFM images are indeterminate, the SEM images reveal a decrease in surface roughness (plan view), and an increase in density (cross-section view) for the film produced at low pressure and subjected to glow discharge treatment.

(a)

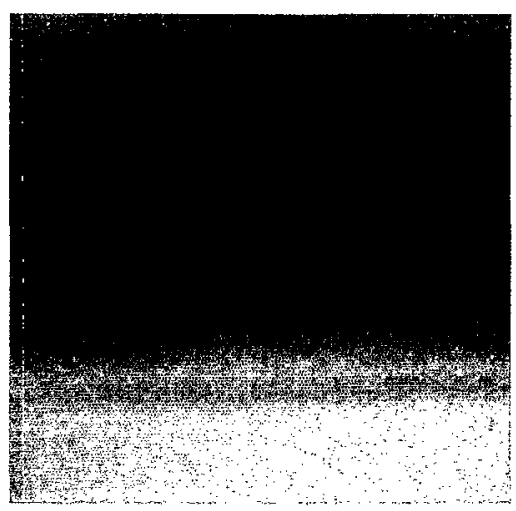

(b)

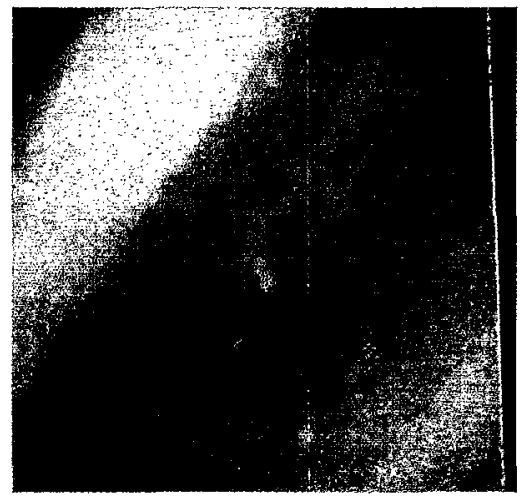

FIGURE 6. AFM Images: (a) High Pressure Coating; (b) Low Pressure Coating

\section{Measurements of Outgassing Rates}

The outgassing rates of the stainless steel chambers with and without TiN coating have been measured using the orifice method. A schematic of the outgassing setup is shown in Figure 7. The orifice size is $3.2 \mathrm{~mm}$ in all cases. Outgassing measurements were made with a $2701 / \mathrm{s}$ sputter-ion pump and TSP pump with a base pressure of $<10^{-11}$ Torr and speed of $1000 \mathrm{l} / \mathrm{s}$ for $\mathrm{H}_{2}$. Inverted magnetron gauges, with a gauge correction factor of 2.4 for $\mathrm{H}_{2}$ were placed on each side of the orifice, while a quadrupole residual gas analyzer was placed on the pump side of the orifice. To determine the hydrogen outgassing rate, chambers were subjected to a 48 hour $250^{\circ} \mathrm{C}$ in-situ bake to remove water vapor and surface gases. Outgassing rates for typical chambers coated under a variety of conditions, are compared to an uncoated chamber and summarized in Table 2.

TABLE 2. Hydrogen Outgassing Rates for SNS Vacuum Chambers

\begin{tabular}{ccc}
\hline Chamber \# & Q (Torr-1/s $\left.\mathbf{~ c m}^{2}\right)$ & Comments \\
\hline$\# 2 \mathrm{~A}$ & $2.5 \mathrm{E}-13$ & in-situ $250^{\circ} \mathrm{C}$ bake, without \\
& $(120$ hours, post-bake) & TiN coating \\
$\# 3 \mathrm{~A}$ & $2.1 \mathrm{E}-13$ & in-situ $250^{\circ} \mathrm{C}$ bake, with high \\
& $(96$ hours, post-bake $)$ & pressure TiN coating \\
$\# 5 B$ & $1.9 \mathrm{E}-13$ & in-situ $250^{\circ} \mathrm{C}$ bake, with low \\
& $(72$ hours, post-bake) & pressure TiN coating \\
\hline
\end{tabular}




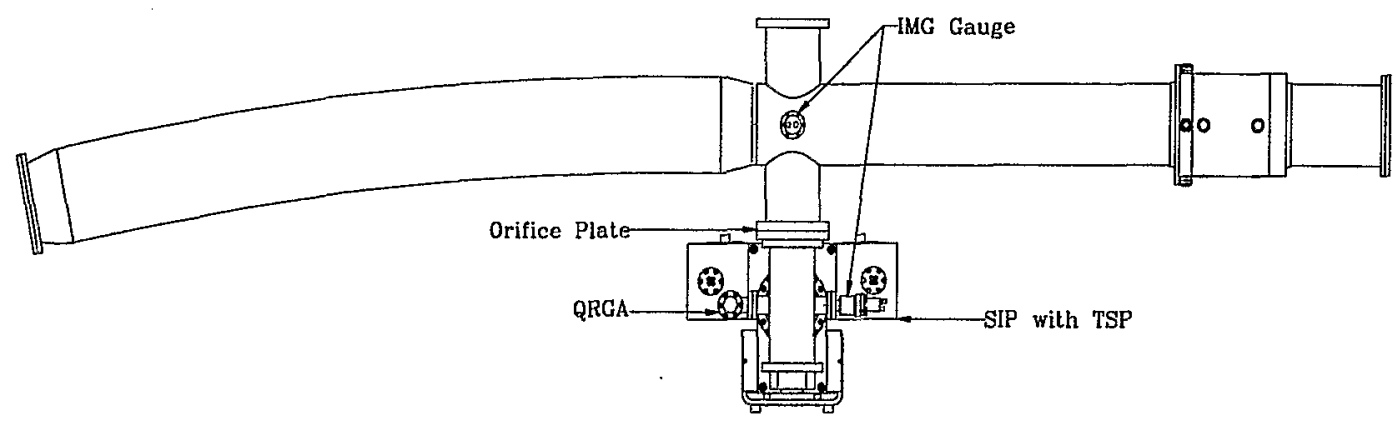

FIGURE 7. Schematics of the outgassing measurement setup using the orifice method.

Saito et-al [1] demonstrated that TiN coating on stainless steel can reduce hydrogen outgassing by two orders of magnitude. Their TiN films were prepared by the hollow cathode discharge-activated reactive evaporation method. Implicit in their findings were a minimum thickness requirement of $1 \mu \mathrm{m}$ as well as stoichiometric films with few defects. Additionally, Saito's substrates were electrolytically polished $304 \mathrm{~L}$ stainless steel whereas our chambers typically have a $2 \mathrm{~b}$ finish, and our TiN coating thickness much less than $0.5 \mu \mathrm{m}$. Clearly, we did not meet any of these conditions; however, our result shows a modest reduction of $25 \%$ over the uncoated chamber. Given this fractional improvement, a statistical result would be desirable. Perhaps a thicker TiN coating would further reduce outgassing? Due to the vast work i nvolved in electro polishing, coating and then making measurements on such a large chamber, these activities are cost prohibitive and unlikely at this juncture.

\section{SUMMARY}

DC magnetron sputtering has been successfully developed to coat the SNS ring vacuum chambers with TiN. This coating also reduced fractionally, the insitu baked $\mathrm{H}_{2}$ outgassing rate by $\sim 25 \%$, showing potential evidence of a permeation barrier to hydrogen diffusion. SEM images reveal film surface structures strongly d ependent on -sputtering pressure and the outgassing rates dependent on the film structure. Further improvement in outgassing may be possible by thicker and denser TiN coatings.

\section{ACKNOWLEDGMENTS}

The authors would like to thank R. Sabatini, J. Warren and M. Lin of Brookhaven for AES, SEM and AFM analysis of some coated coupons, and C-A Vacuum Group in setting up the coating system and the outgassing measurement system. 


\section{REFERENCES}

1. Saito, K., et al., J. Vac. Sci. Technol. A13, 556-561 (1995).

2. Meng, L.J., Azevedo, A. and dos Santos, M.P., Vacuum 46/3, 233-239 (1995).

3. Nah, J.W., et al, J. Vac. Sci. Technol. A17, 463-469 (1999).

4. Hseuh, H.C., Liaw, C.J. and Mapes, M., "Design of SNS Accumulator Ring Vacuum Systems," in Proc. 1999 Particle Accel. Conf., New York, pp1345-1347 (1999).

5. He, P., et al., "Development of TiN Coating for SNS Ring Vacuum Chambers," in Proc. 2001 Particle Accel. Conf., Chicago, USA. pp2159-2161 (2001).

6. Thornton, J.A., J.Vac. Sci. Technol. 11, 666-671 (1974). 